\title{
Drop Size Distribution of Rain Droplet Using Non-Subsampled Contourlet Transform and Double Density Dual tree Transform
}

\author{
P. Ghonge and K. Tuckley \\ Ramrao Adik institude of Technology, Electronics Engineering, Nerul, Navi Mumbai, Maharatra, India \\ \{ghongepa@yahoo.com; kushal_tuckely@rediffmail.com\}
}

\begin{abstract}
This paper deals with algorithm for extraction information from visualized droplets using signal processing. Study show that rain is "patchy" in nature and consists of patches of Drop Size Distributions (DSD) over different scales. The characteristics of high resolution, shift-invariance, and high directionality of Non Subsampled Contourlet Transform (NSCT) and Double-density Dual-tree Discrete Wavelet Transform (DDDWT) are tested. Both transform representing the directional information and capturing intrinsic geometrical structures of the objects. In this paper two transform tested to fuse images and relative parameters are tested to suggest effective transform. Hence, we propose to exploit algorithm to extract information DSD with detail information of rainfall. The proposed methods are tested Rain Images-2 datasets and compared with the standard parameters. Visual and quantitative results demonstrate the efficiency of the proposed methods.
\end{abstract}

Keywords: Drop size distribution; Patchy; Non Subsampled Contourlet Transform; Double-density Dual-tree Discrete Wavelet Transform; Fusion.

\section{Introduction}

The meaning of a waterdrop size distribution is one of those challenges to measure the rainfall in these vibrant environments. In the past the measurement of drop sizes was a straightforward and very laborious task involving shifting and measuring raindrop pellets captured in a box of flour. The most advanced techniques combine different measurements using different instruments each having its own functioning so that, in effect, they are looking at different ensembles of rain patches, at different total drop size distributions[1]. In this article we attempting to provide new application to individual person for extracting information from rainfall by image processing tools, in the process we hope to illuminate the increasing subtlety of the concept of drop size distributions and to develop an awareness of what is really being measured. Image fusion is a process by combining two or more source images from different sensing instruments into a single image with more information. The successful fusion is of great importance in many applications, such as remote sensing, computer vision, medical imaging, servileance and so on [2][3].

The pixel level fusion methods aim to obtain the images with high spatial and spectral resolution simultaneously, the classical fusion methods are principle component analysis (PCA). The PCA method will lose some original spectral features in the process of principal component substitution [4]. In this paper, we applied the Non Subsampled Contourlet Transform (NSCT), which is a shift-invariant version of the contourlet transform. The NSCT is built upon iterated non-subsampled filter banks to obtain a shift-invariant directional multiresolution image representation [5]. The wavelet transform (WT) can preserve spectral information efficiently with property shift-invariance and multi-directionality, the oversampled DWT (discrete wavelet transforms) applied in this paper is the undecimated DWT.

The undecimated DWT is a shift-invariant discrete transform however, the shift-sensitivity of the DWT can be dramatically improved by using a dual-tree DWT, an over complete ex-pansion that is redundant by a factor of 2 [6-7]. The wavelets presented are much smoother than NSCT. The rest of this paper is organized as follows. In Section 2, we present the construction of the NSCT and apply the NSCT to image enhancement, in Section 3 Double Density Dual-tree DWT, in section 4 algorithms and show experimental results in Section 5. Finally, we make conclusions in Section 6.

B. Iyer, S. Nalbalwar and R. Pawade (Eds.)

ICCASP/ICMMD-2016. Advances in Intelligent Systems Research.

Vol. 137, Pp. 767-773.

(C) 2017- The authors. Published by Atlantis Press

This is an open access article under the CC BY-NC license (http://creativecommons.org/licens)es/by-nc/4). 


\section{Non-Subsampled Contourlet Transform}

The non-subsampled contourlet transform combines non subsampled pyramids and non-subsampled DFB's as shown in Figure 1, Non subsampled pyramids provide multiscale de-composition and non-subsampled DFB's provide directional decomposition[2][5]. The non-subsampled contourlet transform achieve the multiscale decomposition, the construct no subsampled pyramids by iterated non subsampled filter banks is shown in Fig. 2 with cascading. For the next level up sample all filters by 2 in both dimensions. Therefore, they also satisfy the perfect reconstruction condition [3] [5].

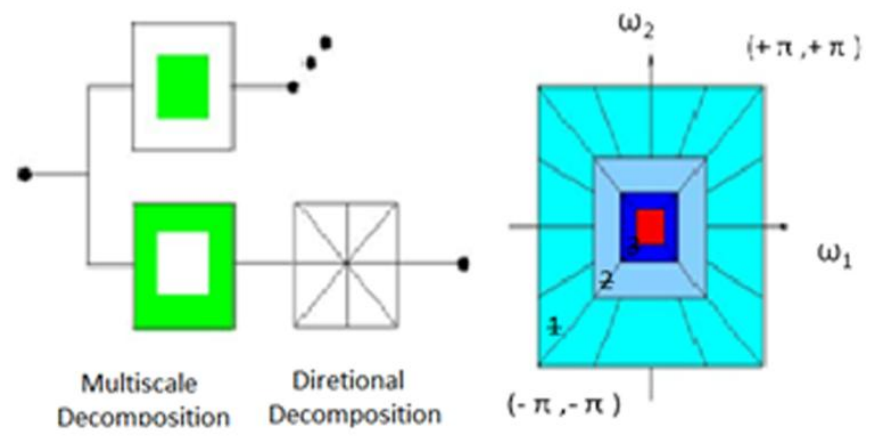

$\begin{array}{ll}\text { (a) Non subsampled pyramid } & \text { (b) Resulting frequency division }\end{array}$

Fig.1. Construction of NSCT

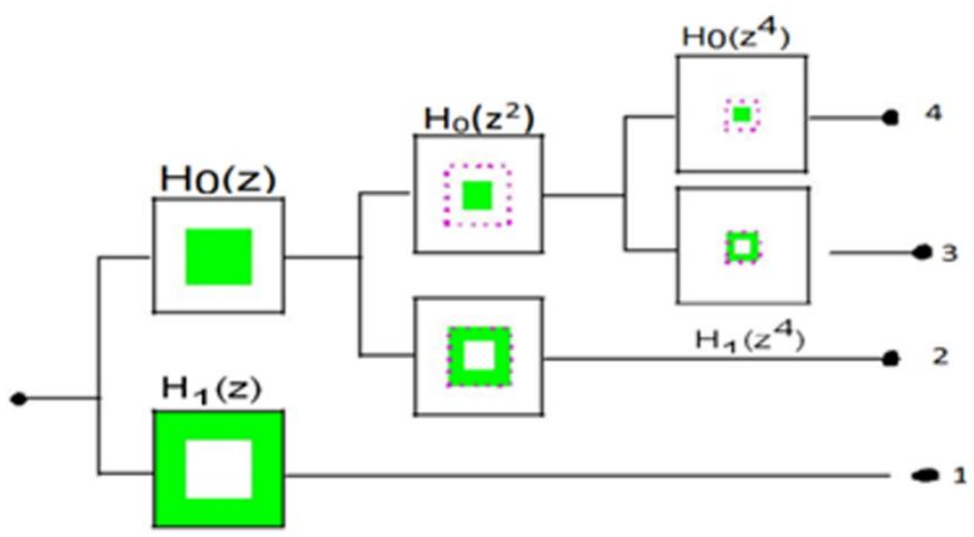

Fig.2. Two-channel non-subsampled filter banks.

The equivalent filters of a $\mathrm{k}$-th level cas-cading nonsubsampled pyramid are given by

$$
H_{n}^{e q}=\left\{\begin{array}{cr}
H 1\left(Z^{2 j^{n+1}} \prod_{j=0}^{n-2} H_{0}\left(z^{2 j}\right)\right. & 1 \leq n<2^{k} \\
\prod_{j=0}^{n-1} H_{0}\left(z^{2 j}\right) & n=2^{k}
\end{array}\right.
$$

Where $z^{j}=\left[z_{1}^{j}, z_{2}^{j}\right]$. These filters achieve multiresolution analysis as shown in Figure 1(a). The NSCT is shiftinvariant such that each pixel of the transform subbands corresponds to that of the original im-age in the same location. Therefore, we gather the geometric information pixel by pixel from the NSCT coefficients.

\section{Double Density Dual-tree Discrete wavelet Transform}

The dual-tree DWT is based on concatenating two critically sampled DWTs. The filterbank structure corresponding to the dual-tree DWT simply consists of two critically sampled iterated filterbanks operating in parallel [6-8].as shown in figure 3.

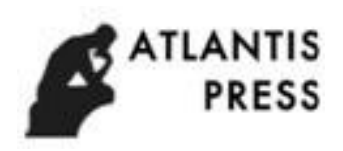




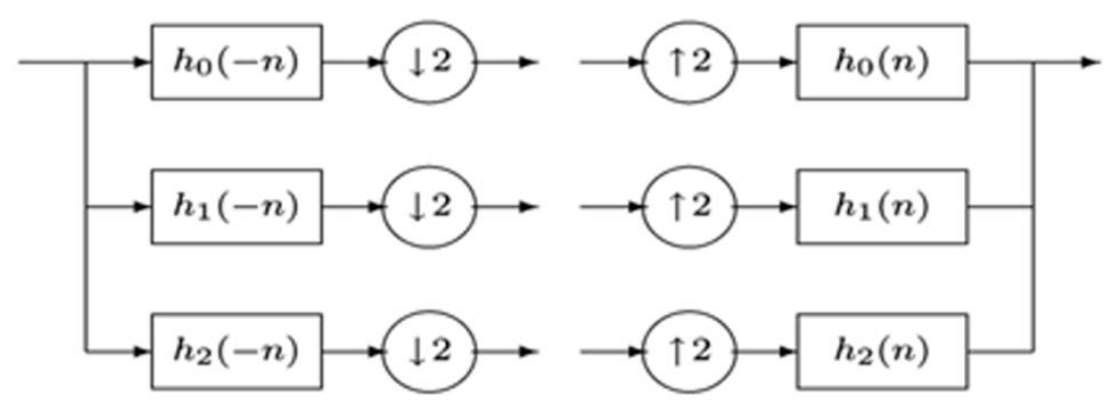

Fig.3. Filterbanks in parallel

The double-density dual-tree DWT proposed on the basis of concatenating two oversampled DWTs. the implementation of the double-density dual-tree [7],

This paper will denote the filters in the first filterbank by $h_{i}(n)$ and the filters in the second filterbank by $g_{i}(n)$ for $i=0,1,2$ all the filter banks to be considered the synthesis filters. The Z transform of $h_{i}(n)$ denoted by $H_{i}(z)$, all the filter coefficients $h_{i}(n)$ and $g_{i}(n)$ are real valued having frequency response $H_{i}\left(e^{j w}\right)$ and $G_{i}\left(e^{j w}\right)$ all satisfy PR conditions [7] [9].
$H_{i}(z)=Z T\left\{h_{i}(n)\right\}=\sum_{n} h_{i}(n) Z^{-n} \quad 2$
$H_{i}\left(e^{j w}\right)=\operatorname{DTFT}\left\{h_{i}(n)\right\}=\sum h_{i}(n) e^{-j n w}$
and

The scaling and wavelet functions are defined implicitly through the dilation and wavelet equations

$$
\begin{array}{lr}
\emptyset h(t)=\sqrt{2} \sum_{n} h o(n) \emptyset h(2 t-n) & 4 \\
\psi_{h, 1}(t)=\sqrt{2} \sum_{n} h_{1}(n) \emptyset h(2 t-n) & 5 \\
\psi_{h, 2}(t)=\sqrt{2} \sum_{n} h 2(n) \emptyset h(2 t-n) & 6 \\
\emptyset_{h}(t)=\sqrt{2 \sum_{n} h_{o}(n) \phi_{h}(2 t-n)} & 7
\end{array}
$$

Discrete-time filters $h_{i}(n)$ and $g_{i}(n)$ define the wavelets, it is necessary to translate the Hilbert transform relations (9) and (10) into constraints to be imposed on the filter bank,

The design of filter for given $K_{i}$ for $\mathrm{i}=0,1,2$, The six filters satisfying a) perfect reconstruction conditions b) Hillbert pair relations c) Zero moment conditions d) shift property, We propose, for the double-density dual-tree DWT that the set of filters take the following form.

$$
\begin{aligned}
& H_{0}(Z)=D(Z)\left(1+Z^{-1}\right)^{k_{0}} Q_{0}(Z) \\
& H_{1}(Z)=-(Z)^{-L} D(-1 / z)\left(1-Z^{-1}\right)^{K_{1}} Q_{1}(Z) \\
& H_{2}(Z)=-(Z)^{-L} D(-1 / z)\left(1-Z^{-1}\right)^{K_{2}} Q_{2}(Z) \\
& G_{0}(Z)=Z^{-L} D(1 / z)\left(1+Z^{-1}\right)^{K_{0}} Q_{0}(Z) \\
& G_{1}(Z)=D(-Z)\left(1-Z^{-1}\right)^{K_{1}} Q_{1}(Z) \\
& G_{2}(Z)=D(-Z)\left(1-Z^{-1}\right)^{K_{2}} Q_{2}(Z)
\end{aligned}
$$

There are four transfer functions $D_{i}(Z)$ and $Q_{i}(Z), i=0,1,2$ which will be determined according to the remaining properties. $D(Z)$ will be determined so that HP relation should satisfy. From [7] [11],[12].we can write

$$
G_{0(z)}=H_{0}(Z) \frac{z^{-L} D(1 / Z)}{D(z)}
$$

We can recall all pass system function with $H_{0}(Z)=1$

$$
A(Z)=\frac{z^{-L} D(1 / z)}{D(Z)}
$$

So that in general we can write $G_{i}(Z)=A(-1 / Z) H_{i}(Z), \quad i=1,2$ 
The transfer function $A(-1 / Z)$ is also all pass

$G_{i}\left(e^{j \omega}\right)=A\left(-e^{-j \omega}\right) H_{i}\left(e^{j \omega}\right)$

$=A\left(e^{j(\omega-\pi)}\right) H_{i}\left(e^{j \omega}\right)$

$=\left(e^{j(\omega-\pi)}\right) H_{i}\left(e^{j \omega}\right)$

Its mean $\theta_{i}(w)=-\theta_{i}(w-\pi)$ fo $i=1,2$

Finding $Q_{i}(z)$ of minimal degree so that the perfect reconstruction conditions are satisfied, assuming that $h_{i}(n)$ and $g_{i}(n)$ have the same autocorrelation function

$H_{i}(\mathcal{Z}) H_{i}(1 / \mathcal{Z})=G_{i}(\mathcal{Z}) G_{i}(1 /(\mathcal{Z})), i=0,1,2$

Above equation satisfy all PR conditions in addition to this

$G_{i}(Z) G_{i}(-1 / Z)=A(Z) A(-1 / Z) H_{i}(Z) H_{i}(1 / Z)$

for $i=0,1,2$ and it becomes

$\sum_{i=0}^{2} G_{i}(Z) G_{i}(-1 / Z)=A(Z) A(-1 / Z) X \sum_{i=0}^{2} H_{i}(Z) H_{i}(-1 / Z)$

If all PR conditions satisfied then product filter can be defined as [6].

$P_{0}(Z)=H_{0}(Z) H_{0}(1 / Z)=G_{0}(Z) G_{0}(1 / Z)$

$=D(Z) D(1 / Z)\left(Z+2+Z^{-1}\right)^{K_{0}} Q_{0}(Z) Q_{0}(1 / Z)$

$=D(\mathcal{Z}) D(1 / \mathcal{Z})\left(\mathcal{Z}+2+\mathcal{Z}^{-1}\right)^{K_{0}} R_{0}(\mathcal{Z})$

Where

$R_{0}(Z):=Q_{0}(Z) Q_{0}(1 / Z)$

The design of wavelets for the DDDWTs described is reliable on filter coefficient optimization to minimize aliasing in a multistage filter bank. The development of the DDDWT motivated by the special properties of complex wavelet transforms.

\section{Image Fusion Algorithm}

The approximation characteristics of an image belongs to the low-frequency part, while the high- frequency counterpart exhibits detailed features of edges .In this article NSCT and DDDWT method is utilized to separate the high and low components of source image in frequency domain, and then the two parts are dealt with the fused image can be reliable for clear and better understood. Here we used Maximum energy algorithm to coefficients which including important features of the two source images. The main function of spatial details as possible as Maximum energy, based on local self-adaptive fusion method is used in band-pass directional sub-band [5]. The fusion process is shown in Figure 4.

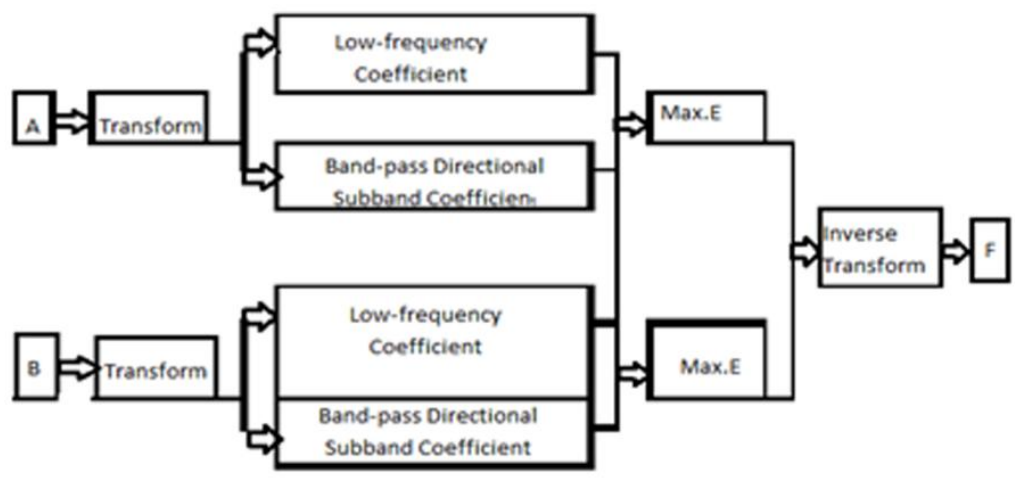

Fig.4. Block diagram of image fusion technique.

In the proposed method of image fusion by the absolute maximum selection fusion rule. Suppose $\mathrm{A}(\mathrm{x}, \mathrm{y})$ and $\mathrm{B}(\mathrm{x}, \mathrm{y})$ are the two images to be fused and their coefficients are $W_{1}(m, n)$ and $W_{2}(m, n)$ respectively, then Absolute Maximum energy.

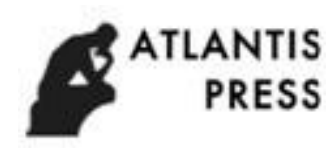




$$
W(m, n)=\left\{\begin{array}{l}
W_{1}(m, n), i f\left|W_{1}(m, n)\right| \geq\left|W_{2}(m, n)\right| \\
W_{2}(m, n), i f\left|W_{2}(m, n)\right|>\left|W_{1}(m, n)\right|
\end{array}\right.
$$

\section{Experimental Results and Analysis}

Experimental data is collected by two different image sensing instruments placed at 90 degree apart having common centre point in restricted three meter square rectangular area. All source images registered with 256 gray levels and are processed under platform MATLAB 2014b. By comparing with typical algorithms below NSCT and DDDWT based maximum selection fusion rule for individual frames of video clip. Below figures gives analysis of algorithms.

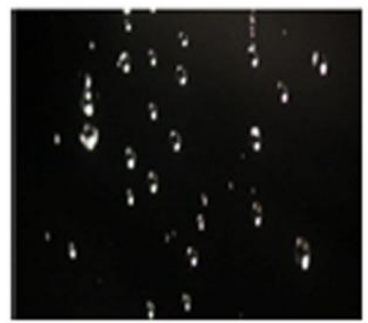

a) Left focus

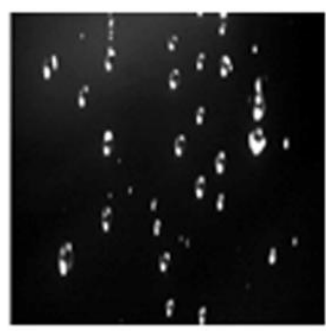

c) DDDWT d) NSCT Fused Image
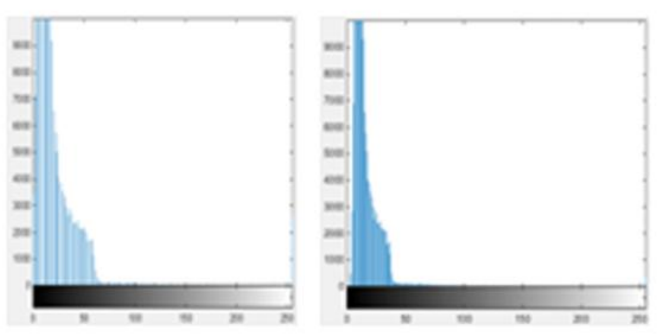

\section{c) DDDWT f) NSCT Histogram}

Fig.4. Performance of Algorithm

The histogram of DDDWT fused image covers wider area than NSCT image, it conclude DDDWT protect edges and give smooth DSD. The performance and quality measurement parameter of fused data are tested, root mean square error (RMSE), standard deviation (SD), percentage residual difference(PRD), Signal to Noise Ratio (NSR), correlation coefficient (CC), relative variance(VR) and quality index (Q). Corresponding fused image parameters and the $\mathrm{Q}$ index. which measures the amount of edge information "transferred" from source images. Here, larger $\mathrm{Q}$ value means better algorithm performance. Comparison of transforms with the fusion method for multi-focus images, DDDWT gives better performance. The below table summarize the performance of parameters and calculate waterdrop sizes with average rainfall for 15 minutes is shown and graphical representation of parameters in figure 6 . 
Table 1. Result in Tabular Form

\begin{tabular}{|c|c|c|c|c|c|c|c|c|c|}
\hline \multirow{2}{*}{$\begin{array}{l}\text { Parameter } \rightarrow \\
\text { Method } \downarrow\end{array}$} & \multirow[t]{2}{*}{$\mathrm{CC}$} & \multirow[t]{2}{*}{ SD } & \multirow[t]{2}{*}{ RMSC } & \multirow[t]{2}{*}{ PRD } & \multirow[t]{2}{*}{ PSNR } & \multirow[t]{2}{*}{$\mathrm{Q}$} & \multicolumn{2}{|c|}{$\begin{array}{c}\text { Drop } \\
\text { size }(\mathrm{mm})\end{array}$} & \multirow[t]{2}{*}{$\begin{array}{l}\text { Avg Rainfall } \\
(15 \mathrm{~mm})\end{array}$} \\
\hline & & & & & & & small & Large & \\
\hline NSCT & 0.98 & 7.38 & 1.16 & 0.04 & 47.43 & 8.23 & 0.264 & 5.26 & 2686.4 \\
\hline DDDWT & 1.00 & 3.70 & 0.95 & 0.01 & 49.56 & 12.73 & 0.646 & 5.99 & 2771.2 \\
\hline Experimental & - & - & - & - & - & - & - & - & 2873.1 \\
\hline
\end{tabular}

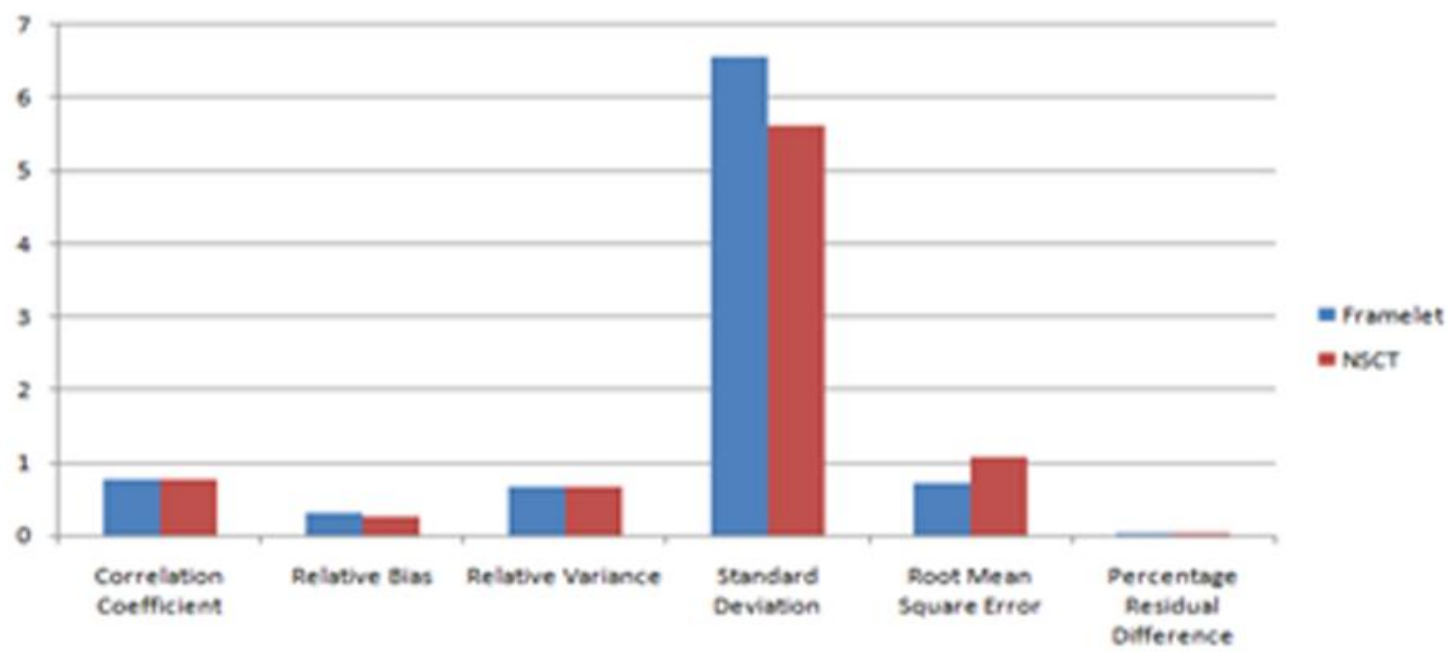

Fig.4. Graphical comparisons of Parameters .

This System compared with the existing techniques over rainfall data to get the characteristics and variability of the drop size distribution (DSD) and related information . By using advance image processing tools the direction of rain fall and rain fall rate can be measured. It provides better alternatives to measure the rainfall technique having numerous advantage,. The experiments are conducted at "Regional Metrological Centre" Coloba Mumbai. We had choose the experiments and demonstrate the results. Its observed that our algorithm is more advantageous in all existing system.

\section{Conclusion}

The Drop size distributions of water droplets provide an improved spatial and temporal characterization of the rain phenomena. In this paper, we presented image processing as a new technique for extracting information from rainfall, developed a fully shift-invariant version and high directionality transforms. First NSCT and DDDWTare tested independently to fuse rain database images to extract useful information's. The fusion relative parameters summaries DDDWT is more efficient and helpful image processing transform to extract information from visualized water droplets. This system would be more help to disaster managements in the city, water dam management, traveler, fisherman and farmers to know rainfall situation around in advance. 


\section{References}

[1] Laws, J. O., and D. A. Parsons, 1943: The relation of raindrop-size to intensity. Trans. Amer. Geophys. Union, 24, 452-460.

[2] M. N. Do and M. Vetterli, "The contourlet transform: An effi-cient directional multiresolution image representation," IEEE Trans. Image Proc., 2005, to appear.

[3] A. L. Cunha, J. Zhou, and M. N. Do, "The nonsubsampled contourlet transform: theory, design and applications," IEEE Trans. Image Proc., submitted, 2005.

[4] M. Patil, B.Iyer and R. Arya, "Performance analysis of PCA and ICA algorithms for facial expression analysis" ASIC (Springer), vol. 439, pp. 965-976, Mar. 2016.

[5] Miloud Chikr El-Mezouar, Kidiyo Kpalma, Nasreddine Taleb, and Joseph Ronsin "A PanSharpening Based on the Non-Subsampled Contourlet Transform: Application to Worldview-2 Imagery"

[6] H. Bolcskei, F. Hlawatsch, and H. G. Feichtinger. Frame-theoretic analysis of oversampled lter banks. IEEE Trans. on Signal Pro-cessing, 46(12):3256\{3268, December 1998.

[7] Ivan W. Selesnick, Member "the double-density dual-tree dwt" ieee transactions on signal processing, vol. 52, no. 5, may 2004.

[8] N. G. Kingsbury. Image processing with complex wavelets. Phil. Trans. Royal Society London A, September 1999.

[9] D. Tay and M. Palaniswami, "Solution to the orthogonal M-channel bandlimited wavelet construction proposition," in Proc. Int. Conf Acoustics, Speech, Signal Processing, Montréal, QC, Canada, May1721, 2004.

[10] I. W. Selesnick, "The design of Hilbert transform pairs of wavelet bases via the flat delay filter," in Proc. IEEE Int. Conf. Acoust., Speech, Signal Process., May 2001.

[11] Kunal Narayan Chaudhury "construction of hilbert transform pairs of wavelet bases and gabor-like transforms ieee transactions on signal processing, vol. 57, no. 9, september 2009.

[12] N. G. Kingsbury, "Shift invariant properties of the dual-tree complex wavelet transform," in Proc. IEEE Int. Conf. Acoustics, Speech, Signal Processing (ICASSP), Mar. 1999, pp. 1221-1224. 